Supporting Information

\title{
Colloidal Single-Layer Quantum Dots with Lateral Confinement Effects on 2D Exciton
}

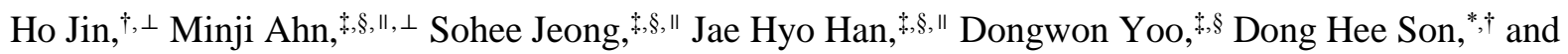
Jinwoo Cheon ${ }^{*}, \sharp,, \|$

${ }^{\dagger}$ Department of Chemistry, Texas A\&M University, College Station, Texas 77843, United States

${ }^{*}$ Center for Nanomedicine, Institute for Basic Science (IBS), Seoul 03722, Korea

${ }^{\S}$ Yonsei-IBS Institute, Yonsei University, Seoul 03722, Korea

"Department of Chemistry, Yonsei University, Seoul 03722, Korea

*dhson@chem.tamu.edu

*jcheon@yonsei.ac.kr 
2H WSe 2 multilayer quantum dots (MQDs)

The binding energies of $\mathrm{W}$ and $\mathrm{Se}$ of $\mathrm{WSe} 2 \mathrm{MQDs}$ (i.e., $\mathrm{W} 4 \mathrm{f}_{5 / 2}(34.4 \mathrm{eV}), \mathrm{W} 4 \mathrm{f}_{7 / 2}(32.3 \mathrm{eV}), \mathrm{Se} 3 \mathrm{~d}_{3 / 2}(55.8$ $\mathrm{eV})$ and $\mathrm{Se} 3 \mathrm{~d}_{5 / 2}(54.9 \mathrm{eV})$ ) measured using X-ray photoelectron spectroscopy (XPS) are consistent with the previously reported values of $2 \mathrm{H} \mathrm{WSe}$.

a

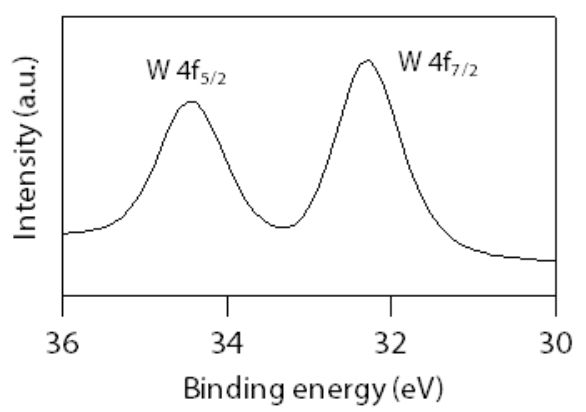

b

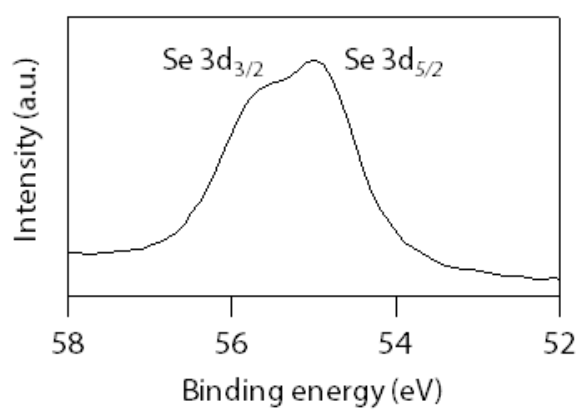

Figure S1. XPS spectra of WSe 2 MQDs. (a) W 4f and (b) Se 3d XPS spectra of $\mathrm{WSe}_{2} \mathrm{MQDs}$. 
Thickness measurement of WSe $\mathrm{C}_{2}$ single-layer quantum dots (SQDs) with various diameters

AFM images of the lateral size-controlled WSe 2 SQDs show that the thickness of each exfoliated quantum dot corresponds to the reported value of single-layer $\mathrm{WSe}_{2}$. The height analysis of more than hundreds of $\mathrm{WSe}_{2} \mathrm{SQDs}$ shows that the average thickness is $\sim 0.7 \mathrm{~nm}$, which confirms that the produced nanoparticles are single layers.
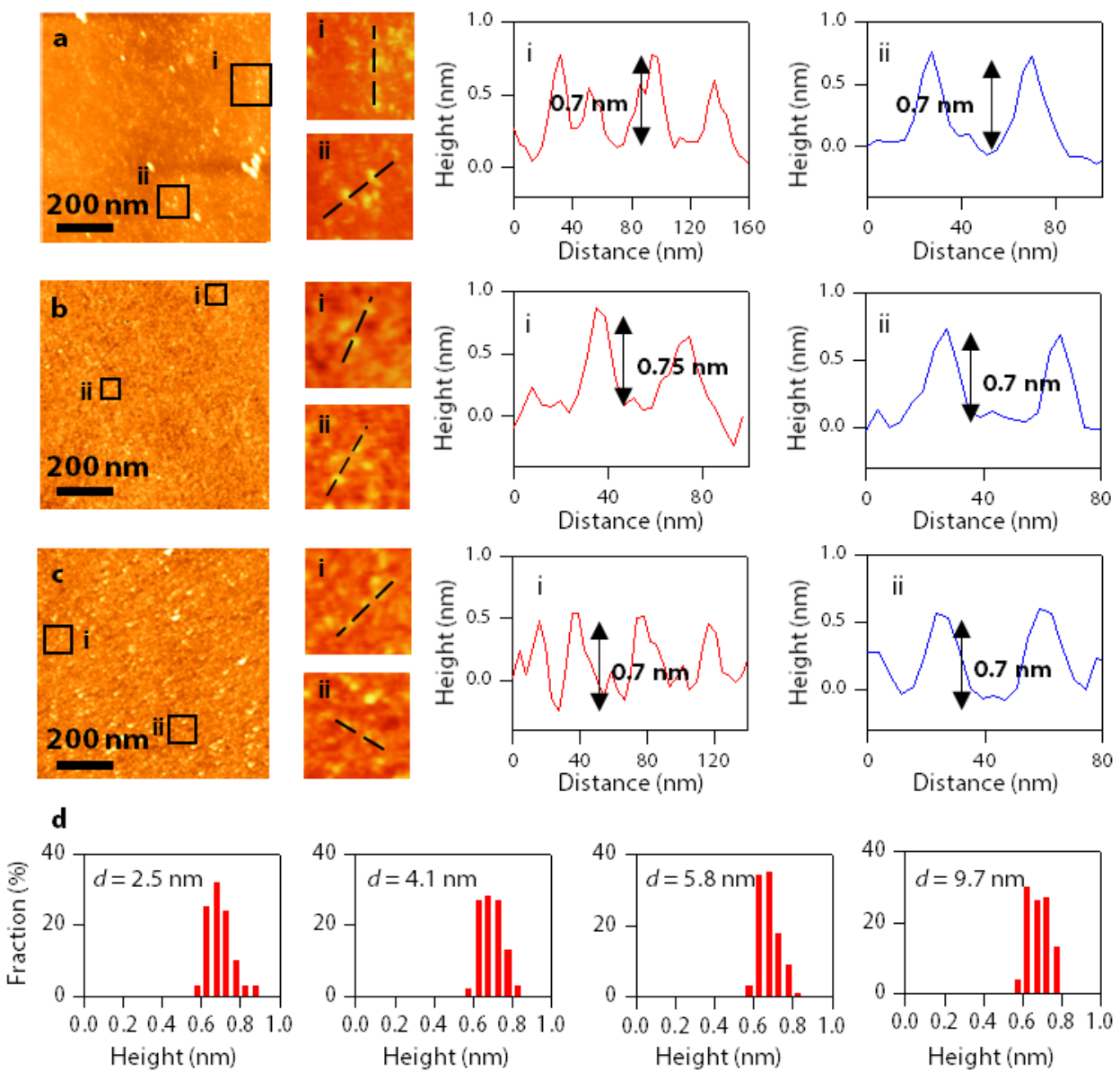

Figure S2. AFM images and statistical analysis of the thickness profile of $\mathrm{WSe}_{2} \mathrm{SQDs}$. AFM images of $\mathrm{WSe}_{2} \mathrm{SQDs}$ with a lateral size of (a) 2.5, (b) 5.8, and (c) $9.7 \mathrm{~nm}$ SQDs. (d) Histograms of thickness for $\mathrm{WSe}_{2} \mathrm{SQDs}$ with $d=2.5,4.1,5.8$, and $9.7 \mathrm{~nm}$, respectively. 


\section{Time-resolved photoluminescence intensity measurement}

Fluorescence lifetime measurements were carried out using a PicoQuant Fluorescence lifetime system (PicoQuant Photonics North America Inc. Westfield, MA USA) equipped with a FluoTime 100 (Compact Fluorescence Lifetime spectrometer), and a PLS450 (sub-nanosecond pulsed LED, center wavelength: $460 \mathrm{~nm}$ ) for measuring lifetimes of ensemble WSe 2 SQD solution.

The acquired time dependent luminescence intensity data was fitted by multi-exponential function, $I(\mathrm{t})=\Sigma \mathrm{a}_{\mathrm{i}} \exp \left(-\mathrm{t} / \tau_{\mathrm{i}}\right)$, where $\mathrm{a}_{\mathrm{i}}$ and $\tau_{\mathrm{i}}$ are the relative amplitude and time constant respectively. Two or three exponential functions were sufficient to fit the data. The averaged luminescence lifetime $(\tau=1 / \mathrm{k})$ was calculated as $\left(\sum \mathrm{a}_{\mathrm{i}} \tau_{\mathrm{i}}\right) /\left(\Sigma \mathrm{a}_{\mathrm{i}} \tau_{\mathrm{i}}^{2}\right)$. All fitting parameters are listed in table $\mathrm{S} 1$.

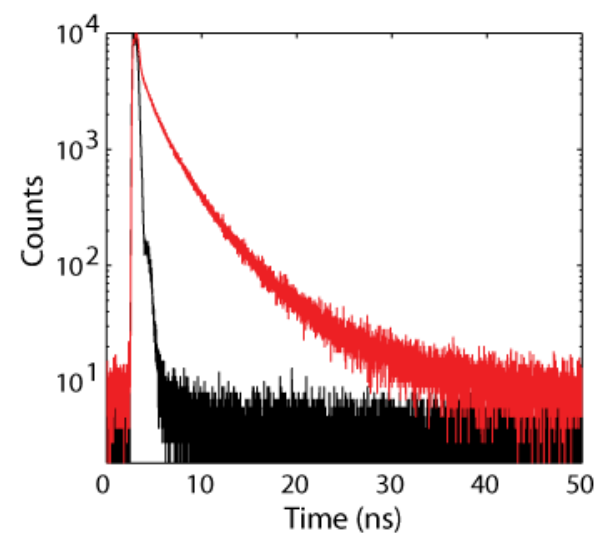

Figure S3. Time-resolved photoluminescence intensity of $\mathrm{WSe}_{2}$ QDs solution (red) and instrument response function (black).

Table S1. Fitting curve of time dependent luminescence intensity data showed in figure $\mathrm{S} 3 . \mathrm{a}_{\mathrm{i}}$ is the relative amplitude for $\tau_{\mathrm{i}}$.

\begin{tabular}{ccccccc}
$\mathbf{a}_{1}$ & $\boldsymbol{\tau}_{\mathbf{1}}(\mathbf{n s})$ & $\mathbf{a}_{2}$ & $\boldsymbol{\tau}_{\mathbf{2}}(\mathbf{n s})$ & $\mathbf{a}_{3}$ & $\boldsymbol{\tau}_{\mathbf{3}}(\mathbf{n s})$ & $\boldsymbol{\tau}_{\text {ave }}(\mathbf{n s})$ \\
\hline 0.34 & 1.2 & 0.56 & 3.0 & 0.10 & 7.2 & 3.8
\end{tabular}




\section{Excitation wavelength dependent PL measurement}

The excitation wavelength dependent PL spectra of the colloidal solution of $\mathrm{WSe}_{2} \mathrm{SQDs}$ are obtained for excitation wavelengths of 320, 350, 370, and $405 \mathrm{~nm}$. The PL peak shifts with the excitation wavelength due to the size heterogeneity of the ensemble sample, as a subset of $\mathrm{WSe}_{2} \mathrm{SQDS}$ is photo-selected at a given excitation wavelength. In contrast, the PL spectra of a single $\mathrm{WSe}_{2}$ SQD exhibit identical PL spectra regardless of the excitation wavelengths.
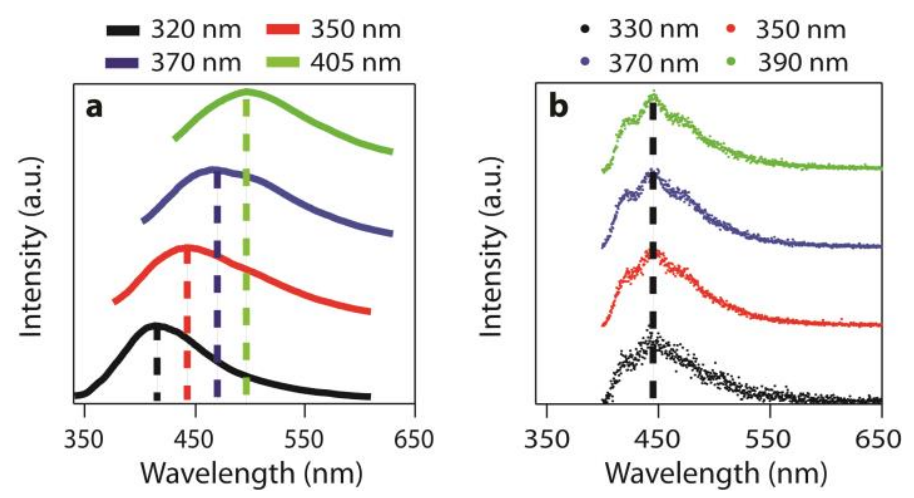

Figure S4. PL measurement of WSe 2 SQDs. (a) Excitation wavelength dependent PL of the $\mathrm{WSe}_{2} \mathrm{SQDs}$ solution. (b) Excitation wavelength independent PL of a single WSe 2 SQD (Figure 4c). The PL spectra are normalized for easier comparison of the peak positions.

Table S2. Stokes shift and full width at half maximum (fwhm) of single-layer $\mathrm{WSe}_{2}$ sheets and $\mathrm{WSe}_{2}$ SQDs
PL (eV)
Stokes shift (eV)
FWHM (eV)

\begin{tabular}{|c|c|c|c|}
\hline Single-layer sheets ${ }^{1}$ & 1.63 & 0.03 & 0.09 \\
\hline SQD-4 & 2.21 & 0.14 & 0.20 \\
\hline SQD-3 & 2.47 & 0.35 & 0.31 \\
\hline SQD-2 & 2.79 & 0.44 & 0.44 \\
\hline SQD-1 & 2.85 & 0.43 & 0.52 \\
\hline
\end{tabular}




\section{Linear polarization anisotropy measurement of the ensemble $\mathrm{WSe}_{2} \mathrm{SQD}$ film}

The linear PL polarization anisotropy of the $\mathrm{WSe}_{2} \mathrm{SQD}$ film is measured using far-field excitation (Figure $\mathrm{S} 4 \mathrm{a})$. A sample is prepared by drop-casting the SQD solution onto a sapphire window. A $405 \mathrm{~nm}$ laser light is used as the excitation source. Initially, the linearly polarized $405 \mathrm{~nm}$ beam is passed through a quarter waveplate to generate circularly polarized light. Subsequently, a linear polarizer is used to vary the linear polarization angle of the excitation light that impinges on the sample at a constant excitation intensity. The PL from the sample is collimated with an achromatic doublet lens, and its polarization is analyzed with a polarizer. The PL spectra are recorded with a CCD spectrometer (QE 65, Ocean Optics) after filtering out the excitation light using a long pass filter.

a

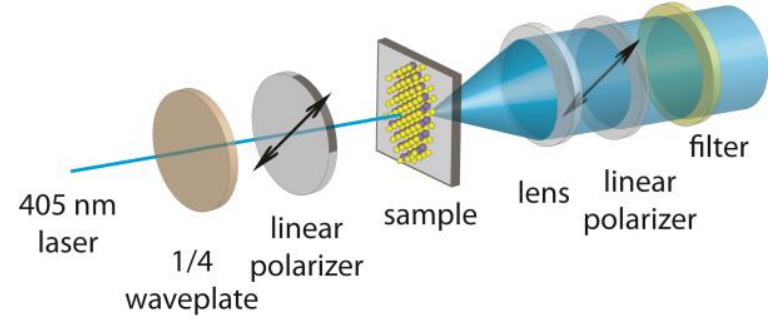

b
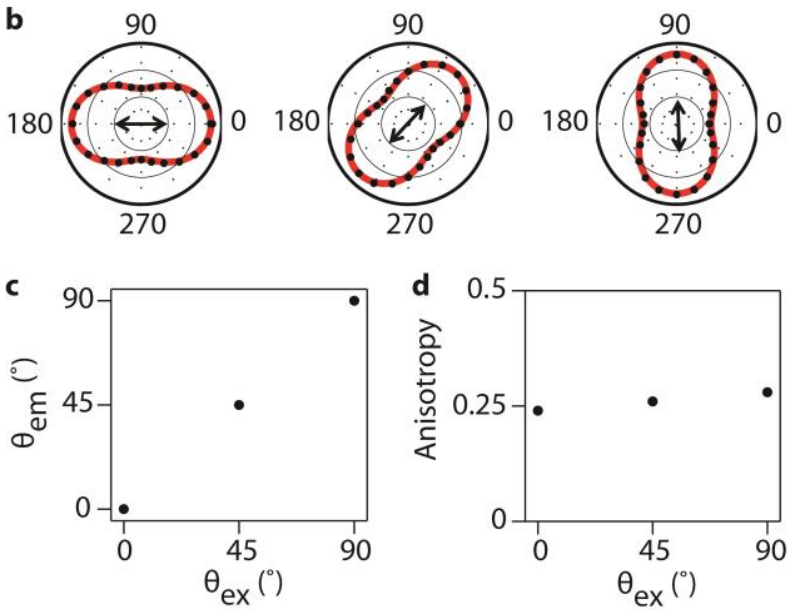

Figure S5. Linear polarization anisotropy measurement. (a) Schematic illustration of polarization anisotropy measurement of an ensemble film sample using linearly polarized far-field excitation. (b) Polar plot of the integrated PL intensity from the ensemble $\mathrm{WSe}_{2} \mathrm{SQD}$ film vs emission polarization angle for the excitation polarization angles of $0^{\circ}, 45^{\circ}$ and $90^{\circ}$. (c) Orientational correlation between excitation polarization angle $\left(\theta_{\mathrm{ex}}\right)$ and emission polarization angle $\left(\theta_{\mathrm{em}}\right)$. (d) PL polarization anisotropy $(r)$ at three different $\theta_{\text {ex. }}$ 


\section{Circular polarization anisotropy measurement of the ensemble WSe $\mathrm{WQD}_{2} \mathrm{SQilm}$}

The film sample is prepared by drop-casting the solution of $\mathrm{WSe}_{2}$ SQDs on a sapphire window. The film on the sapphire window is mounted on an optical cryostat to perform the measurement at $10 \mathrm{~K}$. A $405 \mathrm{~nm}$ laser is used as the excitation light source. The circularly polarized excitation light at $405 \mathrm{~nm}$ was generated by passing the linearly polarized $405 \mathrm{~nm}$ beam through a quarter waveplate. The helicity of the circular excitation is set at $\sigma^{+}$. The emission polarization of $\sigma^{-}$or $\sigma^{+}$is analyzed by a pair of a quarter waveplate and a linear polarizer. The PL spectra are recorded with a CCD spectrometer (QE65, Ocean Optics).

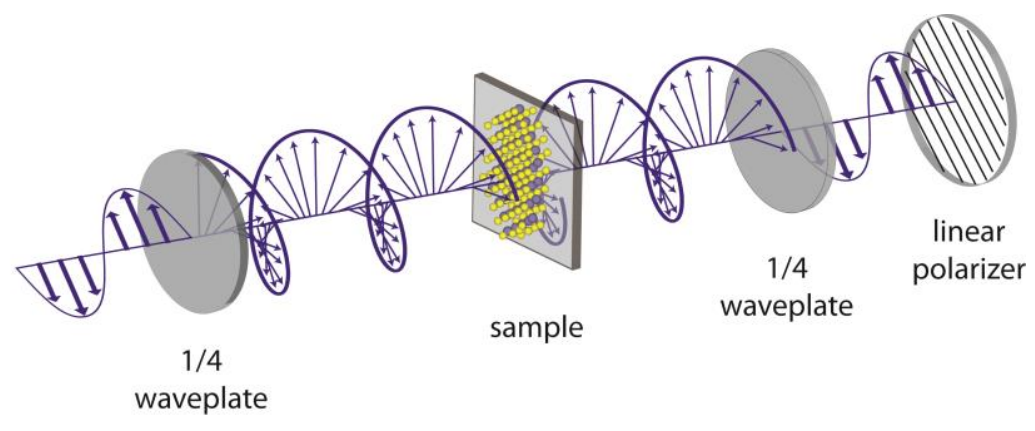

Figure S6. Circular polarization anisotropy measurement. Schematic of the circular polarization anisotropy measurement of the ensemble film sample. 


\section{Note S1. Effective mass approximation}

The bandgap $\left(\mathrm{E}_{\mathrm{g}}\right)$ of a semiconductor increases when its size approaches the exciton Bohr radius. We model a $\mathrm{WSe}_{2} \mathrm{SQD}$ as a cylindrical well with infinite barrier potential. According to the effective-mass approximation, the size dependence of the bandgap can be derived by the following equation. ${ }^{2,3}$

$$
E(a)=E_{g}+\frac{\hbar^{2}}{2}\left(\frac{1}{\mathrm{~m}_{\mathrm{e}}}+\frac{1}{\mathrm{~m}_{\mathrm{h}}}\right)\left(\frac{\mathrm{r}_{\mathrm{nm}}}{a}\right)^{2}
$$

where $\mathrm{E}_{\mathrm{g}}$ is $1.66 \mathrm{eV}$, the direct band gap of monolayer WSe $\mathrm{W}_{2}$ nanosheets ${ }^{1} ; \hbar$ is Plank's constant; $\mathrm{m}_{\mathrm{e}}$ is the effective mass of an electron in a WSe 2 in-plane lattice, $0.26 \mathrm{~m}_{\mathrm{o}} ; \mathrm{m}_{\mathrm{h}}$ is the effective mass of a hole in the $\mathrm{WSe}_{2}$ in-plane lattice, $0.33 \mathrm{~m}_{\mathrm{o}}$, with $\mathrm{m}_{\mathrm{o}}$ being the free-electron mass ${ }^{4} ; a$ is the radius; and $\mathrm{r}_{\mathrm{nm}}$ is the $\mathrm{n}^{\text {th }}$ zero of the Bessel functions of order $\mathrm{m}$. 


\section{Note S2. Evanescent field intensity calculation}

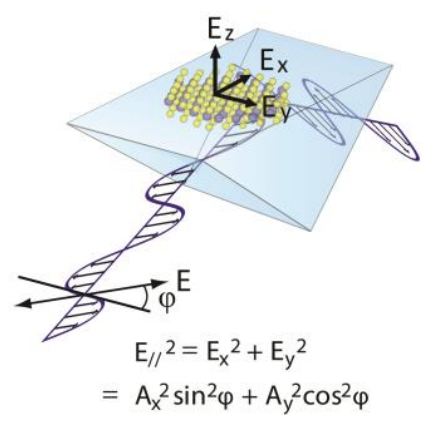

The vector components of the evanescent field $(E)$ in the $\mathrm{x}, \mathrm{y}$, and $\mathrm{z}$ directions at the surface of the prism under total internal reflection are described by the following equation:

$$
E=A_{x} \cos \varphi \exp \left[-i\left(\delta_{P}+\pi / 2\right)\right] \hat{x}+A_{y} \sin \varphi \exp \left(-i \delta_{S}\right) \hat{y}+A_{z} \cos \varphi \exp \left(-i \delta_{P}\right) \hat{z}
$$

where $\varphi$ is the polarization angle of the incident linearly polarized light. $A_{x}, A_{y}, A_{z}$ and the phase lags $\delta_{P}$ and $\delta_{S}$ depend on the incident angle of light at the prism surface and the refractive indices of the prism and its interfacial matrix. ${ }^{5} \mathrm{~A} \mathrm{WSe} \mathrm{S}_{2} \mathrm{SQD}$ is expected to possess an in-plane, 2D-isotropic absorption transition dipole; therefore, it should be excited only by the in-plane components of the evanescent field $\left(\mathrm{E}_{/ /}\right)$. Because the PL intensity is proportional to the number of photons absorbed by the $\mathrm{WSe}_{2} \mathrm{SQD}, \mathrm{E} / /^{2}$ should exhibit a direct 1:1 correlation with the PL intensity. 


\section{References}

1. Huang, J. K.; Pu, J.; Hsu, C. L.; Chiu, M. L.; Juang, Z. Y.; Chang, Y. H.; Chang, W. H.; Iwasa, Y; Takenobu, T.; Li, L. J. ACS Nano 2014, 8, 923-930.

2. Nag, B. R.; Gangopadhyay, S. Phys. Status Solidi A 1993, 179, 463-471.

3. Mallik, K.; Dhami, T. S. Phys. Rev. B 1998, 58, 13055.

4. Shi, H.; Pan, H.; Zhang, Y.; Yakobson, B. I. Phys. Rev. B 2013, 87, 155304.

5. Vacha, M.; Kotani, M. J. Chem. Phys. 2003, 118, 5279-5282. 